\title{
Effet de l'âge et de la compliance du circuit d'éjection sur les courbes de fonction cardiaque et les pressions systoliques du caeur isolé de rat
}

\author{
Effect of age and compliance \\ of the ejection circuit on the cardiac function curves \\ and systolic pressures of an isolated rat heart \\ Claude Sebban*, Irène Wauquier*, Annie Lerède ${ }^{* * *}$, \\ Michel Eugène**, Richard Venet*, Xavier de la Fuente* \\ et Paul Berthaux* \\ ${ }^{*}$ Laboratoire de Biologie du Vieillissement. \\ Faculté de Médecine Pitié-Salpêtrière, Pr. P. Berthaux \\ ${ }^{*}$ C.H.U. Pitié-Salpêtrière, Service de cardiologie, Pr. Grosgogeat \\ ${ }^{* * *}$ Medinat, 45, rue des Saints Pères, Pr. Y. Cherruault
}

Le vieillissement du système cardiovasculaire est caractérisé par deux modifications essentielles :

- une prolongation de la durée d'une contraction concernant la phase de mise en tension et la phase de relaxation [10],

- une altération du tissu conjonctif cardiaque et artériel $[11,17,24]$ modifiant les propriétés mécaniques de ces organes dans le sens d'une rigidité accrue $[8,12$, $15,16]$.

Les conséquences de ces phénomènes, mis en évidence sur des fragments de muscle ou d'artère, sur la fonction pompe du cœur sont encore très mal appréciées. Existe-t-il en effet une altération de cette fonction pompe décrite par la relation entre le débit cardiaque $(\dot{Q})$ et la pression télédiastolique ventriculaire gauche. Dans quelle mesure cette altération est-elle compensée par la régulation de la fonction pompe apportée par le système nerveux autonome? L'accroissement des pressions systoliques (PS) observé avec l'âge chez l'homme et l'animal $[12,14]$ est-il la conséquence des modifications du myocarde, du circuit ou de la régulation nerveuse autonome?

En utilisant dans cette étude le montage du cœur isolé perfusé travaillant décrit initialement par Neely et coll [13], nous faisons abstraction de l'influence du système nerveux autonome. De plus, en modifiant la compliance du circuit d'éjection utilisé, il est possible d'évaluer les effets propres du vieillissement du myocarde par rapport aux modifications du circuit.

Le but de cette étude était de préciser les effets de ces deux paramètres sur la fonction pompe et les pressions systoliques.
Matériel et méthodes

Le montage expérimental est celui du cœur isolé perfusé travaillant décrit par Neelly et coll.

Deux types de circuits dans lequel l'éjection systolique se fait ont été mis au point, de sorte à obtenir, avec le circuit de grande compliance, des courbes de pression dont la morphologie est proche de celle observée en clinique humaine chez l'adulte jeune, et proche de celle du sujet âgé avec le circuit de faible compliance (Fig. 1). Ces deux circuits ne diffèrent que par leur compliance. Ceci a été obtenu en modifiant la quantité d'air contenue dans la chambre d'amortissement, le volume d'eau restant constant. Le volume d'air est de $2 \mathrm{ml}$ et $1,5 \mathrm{ml}$

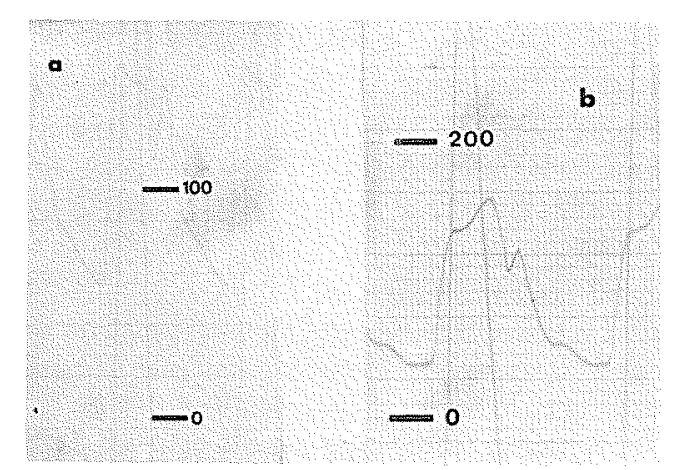

Figure 1 - Morphologie des courbes de pressions obtenues avec chaque circuit chez un animal de 13 mois pour une pression de perfusion de l'oreillette gauche de $20 \mathrm{cmH} 20$. (Pression en $\mathrm{mm}$ de $\mathrm{Hg}$ )

a - circuit de grande compliance

$\mathrm{b}$ - circuit de petite compliance. 
respectivement pour les circuits de grande et petite compliance. Il est remarquablement proche du volume estimé par d'autres $(2,3 \mathrm{ml})$ [3] à partir de mesures expérimentales de la compliance du territoire systémique chez le rat.

Cette modification de la compliance du circuit entraine évidemment des modifications de sa fréquence de résonnance. En admettant que le circuit puisse être ramené à une résistance, une inertie et une compliance serie, les fréquences de résonnance calculées sont de l'ordre de $150 \mathrm{~Hz}$ pour les deux compliances utilisées, c'est-à-dire très supérieures aux fréquences composant les signaux étudiés (fondamentale environ $3 \mathrm{~Hz}$.)

La mise au point des circuits a été réalisée chez des animaux de 6 mois.

Cette expérience a porté sur 16 rats Wistars provenant de notre élevage. Ces 16 rats se répartissent en quatre groupes d'âge différent : $4-7-13$ et 17 mois. Chaque groupe comportait 4 animaux répartis par tirage au sort en deux animaux étudiés sur le circuit de petite compliance et deux sur le circuit de grande compliance. Tous les animaux étaient de sexe masculin.

Après anesthésie à l'éther, le cœur est prélevé et monté sur le circuit selon la technique décrite par Neely et coll. De l'ouverture du thorax au début de la perfusion coronarienne, le temps écoulé a toujours été compris entre $2^{\prime} 25$ et $2^{\prime} 35$. Les mesures hémodynamiques initiales ont été réalisées $15^{\prime}$ après le début de la perfusion coronarienne.

$\mathrm{La}$ pression de perfusion de l'oreillette gauche était initialement de $15 \mathrm{~cm} \mathrm{H}_{2} \mathrm{O}$ amenée successivement à 20.25 et $30 \mathrm{~cm} \mathrm{H}_{2} \mathrm{O}$.

Les déterminations hémodynamiques à chacune des hauteurs ont été effectuées de la deuxième à la troisième minute suivant l'installation de chaque nouvelle pression de perfusion auriculaire. Au total pour un cœur, la durée de l'expérience a été de 30 minutes.

La solution de perfusion est une solution de Krebs Henseleit, équilibrée avec un mélange gazeux $\left(\mathrm{O}_{2}: 95 \%\right.$ $\mathrm{CO}_{2}: 5 \%$ )

(concentration en milli-moles :

$\mathrm{NaCl}: 118-\mathrm{KCL}: 4,7-\mathrm{CaCl}_{2}: 2,5-\mathrm{MgSO}_{4}: 1,2-$ $\left.\mathrm{KH}_{2} \mathrm{PO}_{4}: 12-\mathrm{NaHCO}_{3}: 25\right)$

La température fixée à $37^{\circ}$ a été contrôlée en permanence. Les débits aortique et coronarien ont été mesurés comme dans l'expérience de Neelly.

La mesure des pressions d'éjection a été réalisée par branchement latéral, à $2 \mathrm{~cm}$ de la racine de l'aorte, d'un capteur de pression (Statham $\mathrm{P} 23 \mathrm{DB}$ ). Un raccord rigide de $2 \mathrm{~cm}$ entre le capteur et le point de branchement a été nécessaire.

Le signal ainsi recueilli a été amplifié (HP $8805 \mathrm{C})$ et enregistré (Visicorder Honeywell) à une vitesse de déroulement de $100 \mathrm{~mm} / \mathrm{s}$. Sur la courbe obtenue, la durée du cycle cardiaque, le temps d'éjection, la durée de la diastole et les pressions systoliques ont été mesurés, la valeur considérée dans l'analyse étant une moyenne de cinq déterminations. La durée du cycle cardiaque a été déterminée en prenant comme repère le début de la montée rapide de l'onde de pression, le temps d'éjection a été déterminé par le temps écoulé entre le début de la montée de pression et le fond de l'incisure catacrote. La durée de la diastole a été obtenue par différence PC - TE.

L'analyse des résultats a été réalisée sur ordinateur (IBM Call 360, Statistical Package). Une analyse de la variance a été effectuée (test $F$ de Fischer).

\section{Résultats}

A - Durée du cycle cardiaque - Temps d'éjection - Durée de la diastole :

\section{Durée du cycle cardiaque}

La durée du cycle cardiaque augmente significativement avec l'âge : $F_{41}^{3}=25 p<0,001$

La durée du cycle cardiaque augmente significative: ment quand la compliance du circuit d'éjection diminue: $F_{41}^{1}=16,29 p<0,001$. Ces deux influences sur la durée du cycle cardiaque sont clairement visibles sur la figure 2 .

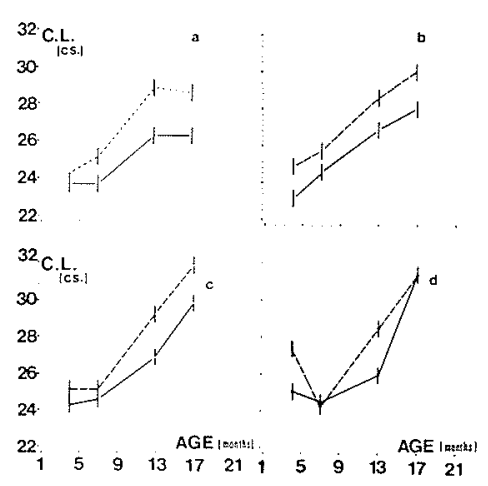

Figure 2 - Relation entre la durée du cycle (CL en cs) et l'âge de l'animal (en mois).

- en traits pleins : circuit de grande compliance

- en tirets : circuit de petite compliance.

La pression de perfusion de l'oreillette gauche :

a $-15 \mathrm{cmH} 20$

b $-20 \mathrm{cmH} 20$

c $-25 \mathrm{cmH} 20$

$\mathrm{d}-30 \mathrm{cmH} 20$

\section{Temps d'éjection}

Le temps d'éjection ventriculaire gauche augmente significativement quand la compliance du circuit d'éjection diminue : $F_{41}^{1}=81,3 \quad p<0,001$.

L'âge entraine des variations significatives du temps d'éjection : $F_{41}^{3}=13,83 p<0,001$. Ces variations ne sont systématiques que pour les expériences réalisées sur le circuit de faible compliance. Il existe en effet une interaction significative entre les effets de l'âge et de la compliance du circuit d'éjection sur le temps d'éjection: $F_{41}^{3}=13,2 \quad p<0,001$. En définitif, le temps d'éjection augmente avec l'âge dans les expériences réalisées sur le circuit de faible compliance $(r=0,75 p<0,01$, $r=0,92 p<0,01, r=0,82 p<0,01, r=0,75 p<0,05$ 


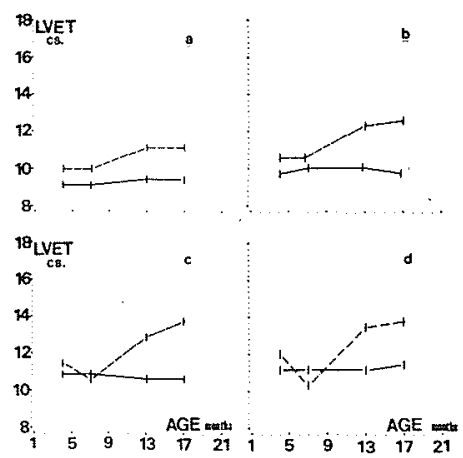

Figure 3 - Relation entre le temps d'éjection ventriculaire gauche (LVET en cs) et l'âge de l'animal (en mois).

- en traits pleins : circuit de grande compliance

- en tirets : circuit de petite compliance

Pression de perfusion de l'oreillette gauche :

a $-15 \mathrm{cmH} 20 \quad \mathrm{c}-25 \mathrm{cmH} 20$

$\mathrm{b}-20 \mathrm{cmH} 20 \quad \mathrm{~d}-30 \mathrm{cmH} 20$

respectivement pour la relation âge $\mathrm{x}$ temps d'éjection à $15,20,25$ et $30 \mathrm{~cm} \mathrm{H}_{2} \mathrm{O}$ de pression de perfusion de l'oreillette gauche). L'ensemble de ces faits est illustré par la figure 3 .

\section{Durée de la diastole}

L'âge entraine des variations significatives de la durée de la diastole : $F_{41}^{3}=33,57 p<0,001$. Quand l'âge augmente, la durée de la diastole augmente et ceci quelle que soit la compliance du circuit d'éjection. Ce fait est illustré par la figure 4 . Cette relation entre l'âge et la durée de la diastole explique pour une part la relation entre âge et fréquence cardiaque.

Les modifications de la compliance du circuit d'éjection n'entrainent pas de variation significative de la durée de la diastole : $F_{41}^{1}=0,29$ N.S.

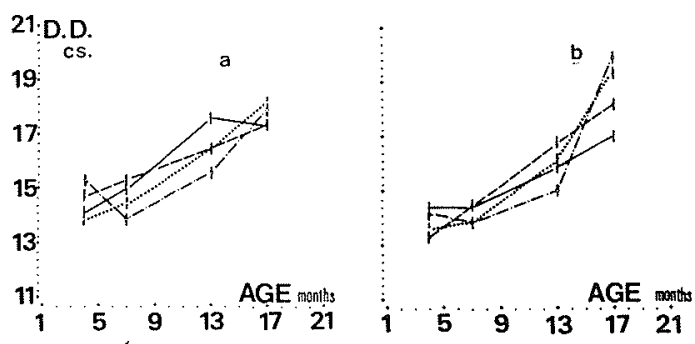

Figure 4 - Relation entre la durée de la diastole (DD en cs) et l'âge de l'animal (en mois).

Pression de perfusion de l'oreillette gauche :

"__ $15 \mathrm{cmH} 20$, "__ _ $20 \mathrm{cmH} 20$, "....." $25 \mathrm{cmH} 20$, " $30 \mathrm{cmH} 20$.

a - circuit de petite compliance

b - circuit de grande compliance.

\section{B - Courbes de fonction cardiaque}

La diminution de compliance du circuit d'éjection entraine une diminution significative du débit cardiaque: $F_{41}^{1}=63,7 p<0,001$. L'âge entraine des variations significatives du débit cardiaque : $F_{41}^{3}: 58,8 p<0,001$.

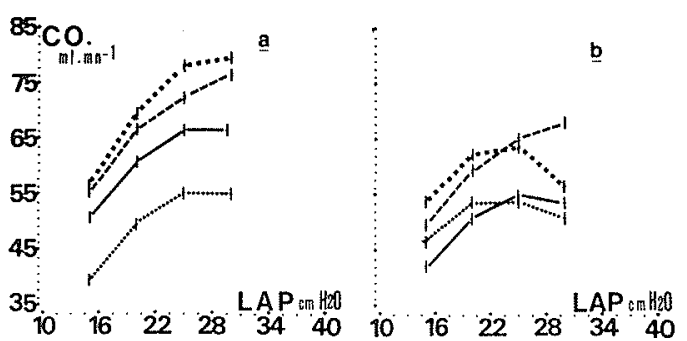

Figure 5 - Effets de l'âge sur les courbes de fonction cardiaque. Débit cardiaque en $\mathrm{Y}(\mathrm{CO}$ en $\mathrm{ml} / \mathrm{mn})$.

Pression de perfusion de l'oreillette gauche en X (LAP en cmH20)

"__ animaux de 4 mois,

"—_ : : animaux de 7 mois,

"-_- " : animaux de 13 mois

"......": animaux de 17 mois

a - circuit de grande compliance,

$\mathrm{b}$ - circuit de petite compliance.

L'étude de la figure 5 montre que pour un circuit de grande compliance, la courbe de fonction cardiaque est déplacée vers le haut de 4 à 7 mois, puis vers le bas, discrètement, de 7 à 13 mois, de façon très importante de 13 à 17 mois Sur le circuit de petite compliance, c'est pour les cœurs de 13 mois que les meilleures performances sont obtenues. A 17 mois, dans ces conditions, les performances sont comparables à celles des cours de 4 mois. Il existe effectivement une interaction significative entre les effets de l'âge et de la compliance sur le débit cardiaque : $F_{41}^{3}=10,4 p<0,001$.

Cette interaction est clairement illustrée par la figure 6. La diminution de la compliance du circuit d'éjection entraine une diminution d'autant plus faible du débit cardiaque que le cour étudié provient d'un animal plus vieux. Les mêmes différences qualitatives sont observées sur les courbes volume d'éjection - pression de l'oreillette gauche. L'âge entraine des variations significatives du volume d'éjection systolique : $F_{41}^{1}=48,4$ $p<0,001$.

La diminution de compliance entraine également des variations significatives du volume d'éjection systolique :

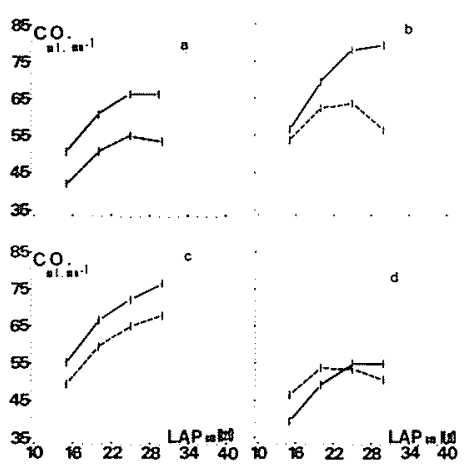

Figure 6 - Effets de la diminution de compliance du circuit d'éjection en fonction de l'âge de l'animal sur les courbes de fonction cardiaque.

Débit cardiaque en Y (CO en $\mathrm{ml} / \mathrm{mn})$

Pression de perfusion de l'oreillette gauche en X (LAP en cmH20)

- en traits pleins : circuit de grande compliance,

en tirets : circuit de petite compliance.

a - animaux de 4 mois, $\quad c$ - animaux de 13 mois,

$\mathrm{b}-$ animaux de 7 mois, $\mathrm{d}-$ animaux de 17 mois. 


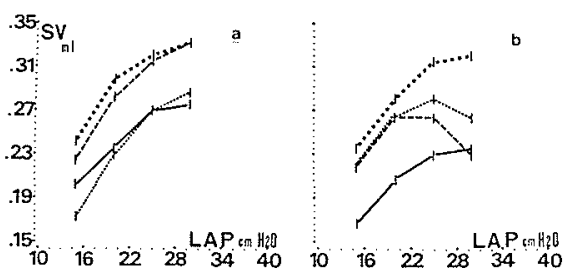

Figure 7 - Effets de l'âge sur les courbes de fonction cardiaque. Volume d'éjection systolique en Y (SV en ml).

Pression de perfusion de l'oreillette gauche en X (LAP en cmH20)

"_ : animaux de 4 mois,

"__ : : animaux de 7 mois,

"_-_ " : animaux de 13 mois,

".......": animaux de 17 mois.

a - circuit de grande compliance,

$\mathrm{b}$ - circuit de petite compliance.

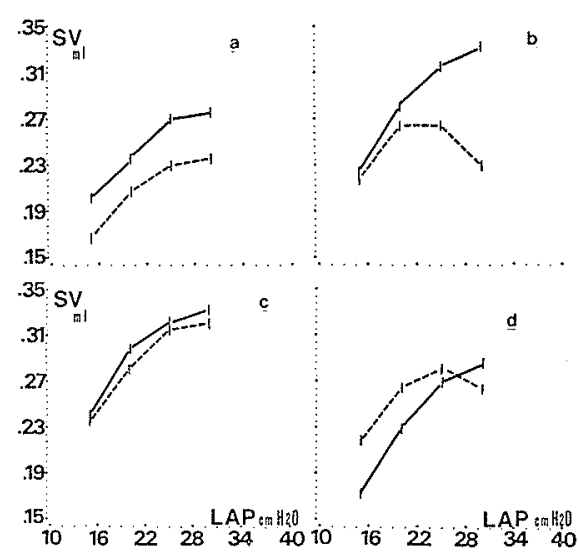

Figure 8 - Effets de la diminution de compliance du circuit d'éjection en fonction de l'âge de l'animal sur les courbes de fonction cardiaque.

Volume d'éjection systolique en $\mathrm{Y}(\mathrm{SV}$ en $\mathrm{ml}$ )

Pression de perfusion de l'oreillette gauche en X (LAP en cmH20)

- en traits pleins : circuit de grande compliance,

. en tirets : circuit de petite compliance.

a - animaux de 4 mois, $\quad c$ - animaux de 13 mois,

$\mathrm{b}$ - animaux de 7 mois, $\quad \mathrm{d}-$ animaux de 17 mois.

$F_{41}^{1}=19,2 p<0,001$. Enfin, il existe aussi une interaction significative entre les effets de l'âge et de la modification de la compliance sur le volume d'éjection systolique : $F_{49}^{1}=11,1 p<0,001$. Les figures 7 et 8 illustrent l'ensemble de ces résultats.

\section{$\mathrm{C}-$ Pressions systoliques}

L'évolution des pressions systoliques aux différentes pressions de perfusion, en fonction de l'âge du myocarde et de la compliance du circuit d'éjection est décrite dans la figure 9.

Dans la plupart des cas la diminution de compliance s'accompagne d'un accroissement de $P S$ significatif $\left(F_{8}^{1}=1,98 N S, F_{8}^{1}=19,52 p<0,01, F_{8}^{1}=24 p<0,01\right.$ et $F_{8}{ }^{1}=5,46 p<0,05$ respectivement pour $15,20,25$ et $30 \mathrm{~cm} \mathrm{H} \mathrm{H}_{2} \mathrm{O}$ de pression de perfusion de l'oreillette gauche).

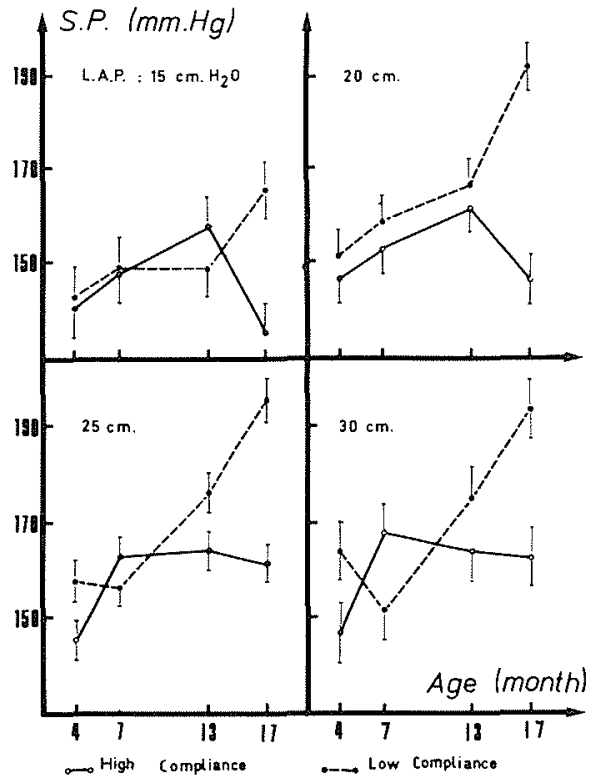

Figure 9 - Evolution de la pression systolique (SP) en fonction de l'âge et de la compliance du circuit d'éjection aux différentes pressions de perfusion de l'oreillette gauche.

L'âge, indépendamment de la compliance, entraine des modifications significatives des pressions systoliques $\left(F_{8}^{3}=1,28 N S, F_{8}^{3}=6,5 p<0,025, F_{8}^{3}=19,26\right.$ $p<0,001, F_{8}^{3}=4,9 p<0,05$ respectivement pour 15 , 20,25 et $30 \mathrm{~cm} \mathrm{H}_{2} \mathrm{O}$ de pression de perfusion de l'oreillette gauche.

Cet effet est surtout net sur le circuit de petite compliance (Fig. 9). Avec ce circuit, les pressions systoliques augmentent régulièrement avec l'âge. Il existe, effectivement, une interaction significative entre les effets de l'âge et de la compliance du circuit sur la valeur des P.S. $\left(F_{8}^{3}=3,49 N S, F_{8}^{3}=8,16 p<0,01, F_{8}^{3}=9,47 p<0,01\right.$, $F_{8}^{3^{8}}=4,57 p<0,05$ respectivement pour $15,20,25$ et $30 \mathrm{~cm} \mathrm{H}_{2} \mathrm{O}$ de pression de perfusion de l'oreillette gauche). Les pressions systoliques augmentent nettement avec l'âge du myocarde seulement sur le circuit de petite compliance. Elles sont pratiquement constantes aux différents âges sur le circuit de grande compliance.

En fait, il est nécessaire de préciser dans quelle mesure l'évolution des P.S. n'est pas simplement la traduction des modifications de débit ou de fréquence observées en fonction de l'âge ou de la compliance du circuit d'éjection.

Effectivement, il existe pour chaque expérience des corrélations très fortes entre P.S. et les paramètres de débit. La plus forte corrélation moyenne est observée entre la P.S. et le V.E.S. $(\bar{r}=0,937)(0,861$ avec le débit). La corrélation avec la fréquence n'est pas significative en moyenne $(\bar{r}=0,657)$.

Pour isoler l'effet propre de la compliance et de l'âge du myocarde sur les $P . S$., nous avons étudié l'effet de ces deux facteurs sur les coefficients de régression, $P S \times$ volume d'éjection systolique, $P S \times$ débit cardiaque, $P S \times$ temps d'éjection ventriculaire gauche calculés pour chaque expérience.

Pour un volume d'éjection ou un débit cardiaque donné, la P.S. est plus importante quand la compliance 
du circuit est faible $\left(F_{11}^{1}=8,78 p<0,05\right.$ et $F_{11}^{1}=6,46$ $p<0,05)$. L'âge entraine également des variations significatives de la P.S. pour un volume d'éjection ou un débit cardiaque donné $\left(F_{11}^{3}=3,7 p<0,05\right.$ et $\left.F_{11}^{3}=4,16 p<0,05\right)$. Les figures 10 et 11 illustrent ces deux effets. La pression systolique pour un volume d'éjection ou un débit cardiaque donné ne varie pas significativement sur le circuit de grande compliance, elle augmente par contre régulièrement avec l'âge sur le circuit de petite compliance.

Pour un temps d'éjection donné, la pression systolique est plus importante pour le circuit de faible compliance avec des myocardes de 7,13 et 17 mois

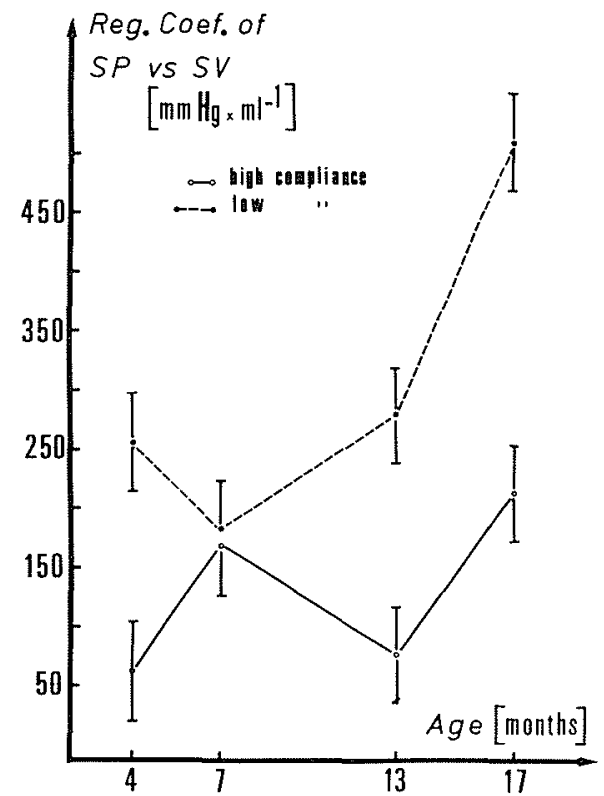

Figure 10 - Influence de l'âge du myocarde et de la compliance du circuit d'éjection sur le coefficient de régression de la pression systolique en fonction du volume d'éjection.

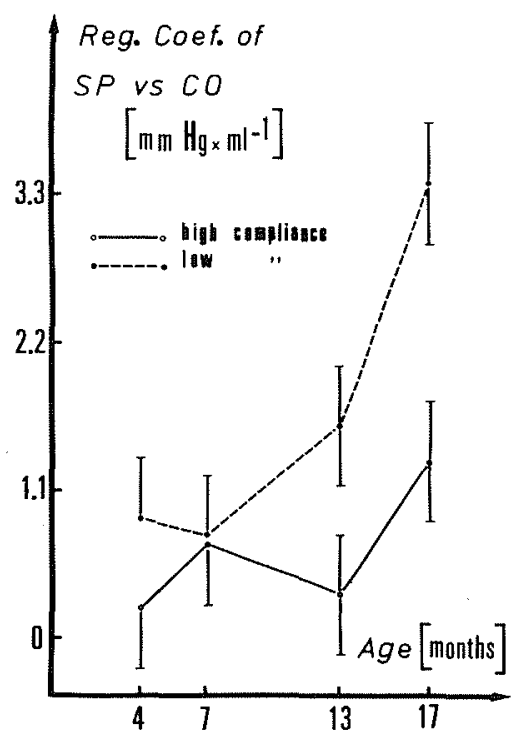

Figure 11 - Influence de l'âge du myocarde et de la compliance du circuit d'éjection sur le coefficient de régression de la pression systolique en fonction du débit cardiaque.

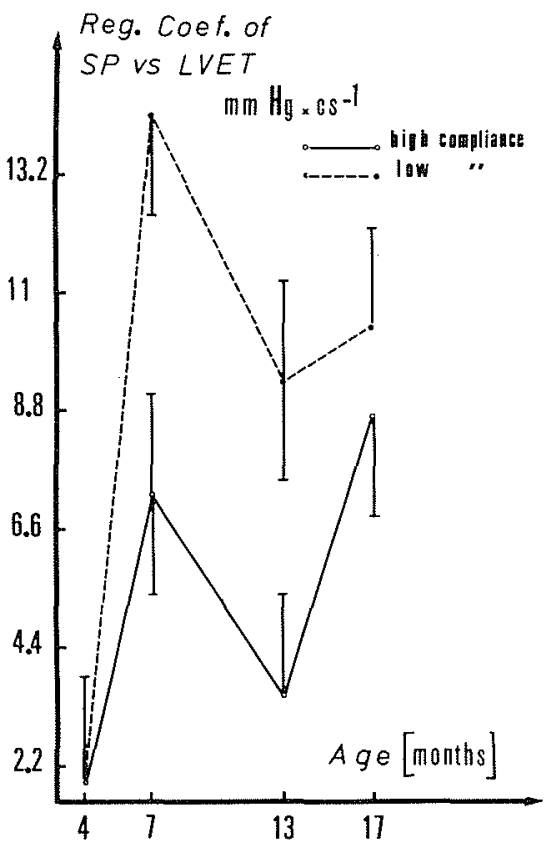

Figure 12 - Influence de l'âge du myocarde et de la compliance du circuit d'éjection sur le coefficient de régression de la pression systolique en fonction du temps d'éjection ventriculaire gauche.

$\left(F_{8}^{1}=5,51 p<0,05\right)$. L'âge entraine également une variation significative de la $P . S$. pour un volume d'ejection donné $\left(F_{8}^{3}=6,32 p<0,05\right)$. En fait, l'examen de la figure 12 montre que cet effet de l'âge ne concerne qu'une différence entre les myocardes de 3 mois et les autres ; à partir de 7 mois la P.S. pour un temps d'éjection donné ne varie pas significativement avec l'âge du myocarde.

\section{Discussion}

\section{Durée du cycle cardiaque}

Chez le rat, les variations de la fréquence cardiaque avec l'âge dépendent des conditions expérimentales : accroissement chez l'animal non anesthésié [18], diminution dans le cas contraire $[5,21]$. Ceci peut être expliqué par une modification avec l'âge de la régulation nerveuse de ce paramètre [9]. L'observation d'une diminution de fréquence cardiaque avec l'âge chez des hommes volontaires ayant reçu atropine et bêta-bloquants [7], si on admet que les effets du système nerveux autonome sont totalement éliminés, peut être expliquée par une modification du myocarde avec l'âge, ou par l'effet des modifications mécaniques du système artériel.

Dans cette étude, nous observons que les deux facteurs interviennent, la fréquence cardiaque diminuant avec l'âge du myocarde et, pour un myocarde d'âge donné, quand la compliance du circuit d'éjection diminue.

L'allongement de la durée du cycle pour un cœur d'âge donné, quand la compliance du circuit dans lequel s'effectue l'éjection diminue, témoigne essentiellement d'un allongement du temps d'éjection. Une relation du même type entre la rigidité artérielle et la fréquence car- 
diaque a été observée dans un groupe de sujets âgés porteurs d'un souffle systolique [4].

L'allongement de la durée du cycle avec l'âge est, dans cette étude, lié essentiellement à un allongement de la durée de la diastole. Il a été observé chez l'homme âgé que la durée de la diastole augmentait significativement quand la compliance ventriculaire diastolique diminuait [20]. Ces deux faits sont peut-être à rapprocher d'autant qu'une diminution avec l'âge de la distensibilité du muscle papillaire de rat a été plusieurs fois rapportée $[6,10,22]$.

La prolongation du $T E$ avec l'âge pour un volume d'éjection donné a déjà été signalée $[19,23]$. Une corrélation positive du $T E$ avec une estimation de la rigidité artérielle a été également observée [19] chez des sujets âgés.

L'observation d'une prolongation avec l'âge du myocarde du temps d'éjection et du temps de relaxation offre un parallélisme surprenant avec la prolongation avec l'âge des temps de contraction et de relaxation du muscle papillaire de rat au cours d'une contraction isométrique [10]. Sur le muscle papillaire, l'origine vraisemblable de ce phénomène siège dans une modification de la récupération du $\mathrm{Ca}$ intracellulaire par le réticulum sarcoplasmique.

Les mêmes observations ont été faites sur le muscle papillaire de rat soumis préalablement à une surcharge systolique ayant induit une hypertrophie myocardique.

La similitude des effets de l'âge et de la diminution de compliance sur la durée du cycle et du temps d'éjection permettent alors de supposer que ce trouble de la recaptation du $\mathrm{Ca}$ du myocarde âgé serait une pérénisation d'un trouble similaire secondaire à l'installation d'une surcharge systolique.

Il reste que si on admet que l'âge où les variations de compliance du circuit d'éjection ont un effet initial respectivement sur la durée de la diastole et celle du temps d'éjection, il est difficile de comprendre par quel intermédiaire cet effet se retrouve au niveau de la fréquence du nœud sinusal. En effet, la régularité presque parfaite des périodes cardiaques ainsi que les fréquences moyennes observées laissent penser, malgré l'absence d'E.C.G., que la commande cardiaque était toujours sinusale.

\section{Fonction pompe du cœur}

Dans cette étude, une altération importante des performances cardiaques est observée avec l'âge. Ces résultats concordent avec les constatations faites chez le rat, non anesthésié [18] et sur des préparations cœurpoumon in situ [21].

La diminution de la compliance du circuit d'éjection entraine également une altération nette des performances cardiaques pour un cœur d'âge donné. Des résultats similaires ont déjà été signalés [3]. Le point le plus important observé dans cette étude est l'existence d'une interaction entre les effets de l'âge du myocarde et de la compliance du circuit d'éjection sur les performances cardiaques.

La diminution de la compliance du circuit d'éjection entraine une altération des performances cardiaques d'autant plus limitée que le cœur étudié est plus âgé. Une meilleure adaptation du myocarde âgé à la diminution de compliance du circuit d'éjection suggère à nouveau que les modifications du muscle cardiaque, survenant au cours du vieillissement entrainent une adaptation des propriétés mécaniques de la pompe cardiaque aux variations de celles du circuit artériel.

\section{Pressions systoliques}

L'observation d'un accroissement des pressions systoliques quand la compliance du circuit d'éjection diminue concorde avec les observations d'autres auteurs. Elle s'accompagne d'une prolongation du temps d'éjection, mais pour un temps d'éjection donné la pression systolique reste significativement plus élevée quand la compliance du circuit diminue.

A l'inverse, l'accroissement des pressions systoliques avec l'âge, pour le circuit de petite compliance, constitue une observation tout à fait originale. Quand on tient compte du temps d'éjection l'âge du myocarde n'a plus d'effet sur la pression systolique.

Il semble donc dans ce deuxième cas que les modifications de P.S. avec l'âge soient dépendantes de celles du T.E. La similitude des figures 3 et 9 est à cet égard extrêmement évocatrice. En fait, ce qui est habituellement qualifié de temps d'éjection décrit précisément l'évolution des pressions artérielles pendant la phase du cycle cardiaque où pressions artérielles et pressions ventriculaires sont pratiquement identiques. Ce temps d'éjection témoigne donc plus précisément du temps pendant lequel la contraction myocardique génère la pression systolique. On peut donc rapprocher les modifications du "temps d'éjection" de la prolongation de la durée de contraction du muscle papillaire avec l'âge. Dans ce cas, l'augmentation de la pression systolique avec l'âge du myocarde, pour le circuit de petite compliance, traduirait la prolongation du temps de contraction ventriculaire. C'est là une hypothèse nouvelle pour expliquer l'accroissement des pressions systoliques observées avec l'âge aussi bien chez l'homme que chez l'animal.

\section{Bibliographie}

[1] BERG B.N., HARMSION C.R. (1955) - Blood pressure and heart size in aging rats. J. Geront., 10, 416-419.

[2] ELIAKIM M., SAPOZNIKOW D., WEINMAN S. (1971) Pulse wave velocity in healthy subjects and in patients with various desease states. Am. Heart $J .82,448-457$.

[3] ELZINGA G., WESTERHOF N. (1973) - Pressure and flow generated by the left ventricle against different impedances. Circ. Res. 32, 178-186.

[4] EUGENE M., SEBBAN C., BERTHAUX P. (1977) - Différences hémodynamiques entre les sujets âgés avec ou sans souffle systolique innocent. Coeur Med, inter., 16, 385-389.

[5] EVERITT A.V. (1958) - The electrocardiogram in ageing male rats. Gerontologia (Basel), 2, 204-212.

[6] GRANATH A., JONSSON B., STRANDELL T. (1970) Physical activity and ageing. Medicine and Sport, Karger, Basl, New-York, 48. 
[7] JOSE A.D., COLLISON B. (1970) - Determinants of the intrinsic heart rate of man. Cardiovasc. Res. 4, 160-167.

[8] KING A.L. (1946) - Pressure volume relation for cylindrical tubes with elastomeric walls : the human aorta. $J$. Appl. Physio. 17, 501-505.

[9] KIRCHHEIM H.R. (1976) - Systemic arterial baroreceptor reflexes. Physiol. Rev., 56, 100-176.

[10] LAKATTA E.G., GERSTENBLITH G., ANGELL C.S., SHOCK N.W., WEISFELDT M.L. (1975) - Prolonged contraction duration in aged myocardium. $J$. Clin. Invest., $55,61-68$

[11] LANSING A. (1959) - The arterial wall : aging, structure and chemistry. Baltimore. Williams and Wilkins 136-160.

[12] LEAROYD B.M., TAYLOR M.G. (1966) - Alterations with age in the viscoelastic properties of human arterial walls. Circ. Res. 18, 278-292.

[13] NEELY F.R., LIEBERMEISTER H., BATTERSBY E.T. MORGAN H.E. (1967) - Effect of pressure development on oxygen consumption by isolated rat heart. Amer. J. Physiol., $212,804-814$

[14] NIELSEN B.J., ROIN J., FABRICIUS S., NIELSEN J.S (1968) - Carotid femoral pulse wave velocity. J. Amer. Geriat. Soc., 16, 658 .

[15] O'ROURKE M.F., BLASZER J.V., MORREELS S.L., KROVETZ J. (1968) - Pressure wave transmission in the human aorta changes with age and in arterial degenerative diseases. Circ. Res. 23, 567-579.
[16] ROACH M.R., BURTON A.C. (1959) - The effect of age on the elasticity of human iliac arteries. Can. J. Biochem. Physio., 37, 557-570.

[17] ROBERT B., ROBERT L., ROBERT A.M. (1974) Elastine, elastase et artériosclérose. Path. Biol. 22, 8, 661-670.

[18] ROTHBAUM D.A., SHAW D.J., ANGELL C.S., SHOCK N.W. (1973) - Cardiac performance in unanesthetized senescent male rat. J. Geront., 28, 287-292.

[19] SEBBAN C., JOB D., DOYON B., BERTHAUX P. (1974) - Souffles systoliques d'éjection et contractilité du ventricule gauche. Rev. Franç. Géront., 4, 36.

[20] SEBBAN C., JOB D., CAEN J.L., DOYON B., PLAS F., BERTHAUX P. (1975) - Ventricular compliance and ageing. Biomedicine 22, 56-61.

[21] SHREINER D.P., WEISFELDT M.L., SHOCK N.W. (1969) - Effect of age, sex and breeding status on the rat heart. Amer. J. Physiol., 217 (1), 176-180.

[22] WEISFELDT M.L., LOEVEN W.A., SHOCK N.W. (1971) - Resting and active mechanical properties of vascular carnae from aged male rats. Amer. J. Physio., 220, 1921-1927.

[23] WILLEMS J.L., ROELANDT J., GEEST H. de, KESTELHOOT H., JOOSSENS J.V. (1970) - The left ventricular ejection time in elderly subjects. Circulation, 17, 37.

[24] WOLINSKY H. (1972) - Long term effects of hypertension on the rat aortic wall and their relation to concurrent aging changes. Morphological and chemical studies. Circ. Res. 30, 301-309.

\section{Discussion}

Le Président remercie M. SEBBAN et ouvre la discussion en souhaitant de nombreuses interventions des "physiciens" à propos de cet exposé très "physiologique".

M. JAFFRIN se montre frappé par la simplicité du fonctionnement du circuit. Il observe cependant que la compliance du système dépend de la pression sanguine et du volume d'éjection et que, pat conséquent, deux coeurs ayant deux volumes d'éjection différents n'auront pas la même compliance.

M. SEBBAN en convient : il se trouve pourtant que l'installation ne fait ainsi qu'accentuer l'effet de l'âge sur les pressions puisque avec l'âge le volume d'éjection diminue.

M. JAFFRIN demande si la compliance du circuit a été calculée et correspond à la compliance artérielle du rat.

M. SEBBAN précise à ce sujet que le calcul a été fait a posteriori mais que le résultat obtenu est en bonne concordance avec - comme équivalent de compliance chez le rat - la valeur de 1,8 millilitres d'air, publiée par d'autres chercheurs.

A propos du calcul de la compliance selon Windkessel, $M$. KOPP insiste sur les difficultés résultant du comportement polytrope du matelas d'air aux fréquences concernées, compliqué par les changements de phase de l'eau à la surface libre. Les deux phénomènes, précise-t-il, sont dépendants de la fréquence. Le deuxième est difficile à contrôler: la compliance réelle est une fonction aléatoire du temps avec un écart-type d'environ $10 \%$. La non-linéarité de Windkessel et de la perte de charge singulière utilisée alourdissent de plus leur exploitation numérique.

Une capacité métallique déformable entièrement remplie de liquide, une ou plusieurs résistances fluides linéaires (capillaires) permettraient une adaptation plus précise et plus maniable au lit vasculaire supposé.
Ceci n'enlève rien à la validité des résultats obtenus in vitro, mais souligne la difficulté classique des expérimentateurs, provenant de la distance entre le faisable et le souhaitable, lorsque l'on souhaite extrapoler un résultat in vitro aux situations réelles.

M. BERGERON, se référant à l'expérience des conduites industrielles, s'étonne que s'agissant de régimes essentiellement variables comme ceux qui sont en cause, l'auteur ne parle pas de la célérité des ondes alors qu'elle varie avec l'élasticité donc avec la compliance.

M. SEBBAN observe que sur son modèle la compliance est strictement limitée à un point précis du circuit et que tout le reste est pratiquement indéformable.

M. JAFFRIN rappelle que, selon les hypothèses de Windkessel, la pression est uniforme partout, donc la célérité infinie.

M. COMOLET indique que la célérité des ondes dans les voies artérielles est de plusieurs mètres par seconde, même chez le rat et que, dans un modèle qui occupe quelques décimètres au maximum, tout le circuit est pratiquement à la même pression au même moment.

M. REMENIERAS remarque à cet égard que le modèle présente une élasticité "concentrée" alors qu'elle est "répartie" dans la réalité. Pour les modèles de conduites industrielles - beaucoup plus rigides - il est possible de répartir cette élasticité en insérant dans les dites conduites un tube souple rempli de gaz.

Pour M. VADOT, la morphologie des ondes de pression obtenue avec une petite compliance évoque celle qui caracté rise un rétrécissement aortique. 
M. SEBBAN en convient. En se référant aux très nombreuses courbes de pression qu'il a enregistrées depuis huit ans sur des sujets âgés, il insiste sur l'importance de la notion de prolongation du temps de contraction associée à cette morphologie.

En réponse à une autre question de M. VADOT, il indique que les conditions de ses essais ne lui permettaient pas d'observer une éventuelle évolution de la durée de la phase isométrique de la contraction systolique.

Le Président rappelle que les expériences présentées par $M$ SEBBAN s'inscrivent dans une longue lignée qui remonte à près d'un siècle et que les procédures - plus récentes - permettant de faire "travailler" le coeur dans diverses conditions de compliance, de résistance, de pression, offrent de nouvelles et intéressantes possibilités aux circuits de cœur isolé actuellement en exploitation. Il se montre surpris de certaines des dispositions adoptées par M. SEBBAN. Il s'étonne en particulier que s'agissant du travail cardiaque, M. SEBBAN parle beaucoup de "débit" et pratiquement pas de "pression".

M. SEBBAN confirme, à cette occasion, que l'objet de ses travaux n'était pas le travail cardiaque mais bien le débit qui conditionne les quantités d'oxygène apportées en tissu et dont l'influence est essentielle dans la pathologie cardiovasculaire du sujet âgé à laquelle se rattachaient ses travaux.
Le Président transmet à M. SEBBAN une question de M. LE PAILLEUR concernant la compliance des ventricules.

M. SEBBAN indique que ce problème est particulièrement difficile, qu'il n'a pas été traité sur les cœurs isolés de ses expériences mais qu'il est en cours d'étude. Le fait que le volume dyastolique du ventricule varie en fonction de l'âge ne signifie pas, selon lui, que la compliance varie en fonction de l'âge.

Sur une question du Président, il précise que les anomalies de volume dyastolique observées chez les sujets âgés ou dan certaines cardiopathies ne traduisent pas forcément un étirement différent des fibres, et donc des conditions de starling différentes.

Le Président remarque en conclusion que la compliance ne doit pas toujours être considérée isolément de tout le cycle cardiaque. Ce qui est significatif c'est la relation pression/volume instantanée, suivie tout au long du cycle.

Il signale les mesures continues de volume ventriculaire menées par SUGA, ainsi que les mesures de débit d'éjection du ventricule enregistrées dans son laboratoire : en remontant à la courbe de la relation pression/volume, on obtient l'élastance - ou la compliance - de façon continue.

Cette orientation est, à ses yeux, une ouverture intéressante sur l'étude de la pompe cardiaque. 